\title{
Generation of Attractors of Rossler Systems with Feedback
}

\author{
Zhihua Huang \& Yali Dong \\ School of Science, Tianjin Polytechnic University, Tianjin 300160, China \\ Tel: 86-22-24584562_E-mail: huangzhihua2184632@163.com
}

\begin{abstract}
In this paper, the problem of attractors is investigated, which are generated by controlled Rossler systems with periodic and bounded feedback functions. These attractors have overlapped "rectangular" form. And the shapes and sizes of generated attractors can be adjusted easily by changing specific system parameters.
\end{abstract}

Keywords: Attractors, Controlled Rossler System, Chaos generation

\section{Introduction}

During the last several decades, nonlinear dynamics and chaos have been taken much attention and various methods have been introduced to investigate the problems (Brown \& Chua, 1996, p.219-249.Gubckenheimer \& Holmes, 1983). After getting the knowledge of underlying mechanism of some complex behaviors such as chaos, people began to consider the control and synchronization to reduce the complexity if possible (Chen \& Yu, 2003. Chen, Zhan \& Hong, 2006), and consider the generation and employment of complex behaviors. Recently, many effective methods have been proposed to generate specific chaotic attractors (Heagy, Platt \& Hammel, 1994, p. 1140-1150), such as multi-scroll attractors (Hwang, Gao \& Liu, 2000, p. 5162-5170), and striped attractors (Chen, Hong, Chen \& Zhong, 2006, p.195-200).

The objective of this paper is to generate a special attractor so that it could meet something special in industry. Rossler attractors are generated by the following Rossler systems:

$$
\left\{\begin{array}{l}
x^{\prime}=-y-z \\
y^{\prime}=x+a y \\
z^{\prime}=z(x-b)+c
\end{array}\right.
$$

Where $a, b, c$ are constant. In (Chen, Hong, Chen \& Zhong, 2006, p.195-200), a new striped chaotic orbit is generated by Rossler systems with additive noise. But the feedback functions in (Chen, Hong, Chen \& Zhong, 2006, p.195-200) are not continuous functions.

In this paper, we will investigate a new Rossler system, which could generate striped chaotic orbits. In contrast to (Chen, Hong, Chen \& Zhong, 2006, p.195-200), the feedback functions can be extend to continuous functions.

\section{Attractors}

Considering the following Rossler system:

$$
\left\{\begin{array}{l}
x^{\prime}=-y-z+l_{1}\left(\alpha_{1}+f_{1}\left(e_{1} x\right)\right) \\
y^{\prime}=x+a y+l_{2}\left(\alpha_{2}+f_{2}\left(e_{2} y\right)\right) \\
z^{\prime}=z(x-b)+c+l_{3}\left(\alpha_{3}+f_{3}\left(e_{3} z\right)\right)
\end{array}\right.
$$

where $f_{1}\left(e_{1} x\right), f_{2}\left(e_{2} y\right), f_{3}\left(e_{3} z\right)$ are bounded and periodical functions. $e_{i}>0,(i=1,2,3)$ is the frequency of $f_{1}\left(e_{1} x\right), f_{2}\left(e_{2} y\right) \quad, f_{3}\left(e_{3} z\right) . \alpha_{i},(i=1,2,3) \quad$ is $\quad$ some given constant. Suppose $m_{1} \leq f_{1}\left(e_{1} x\right) \leq n_{1}, m_{2} \leq f_{2}\left(e_{2} y\right) \leq n_{2}, m_{3} \leq f_{3}\left(e_{3} z\right) \leq n_{3}$. Apparently, $l_{1}\left(\alpha_{1}+f_{1}\left(e_{1} x\right)\right)$ is in $\left[l_{1}\left(\alpha_{1}+m_{1}\right), l_{1}\left(\alpha_{1}+n_{1}\right)\right]$. $l_{2}\left(\alpha_{1}+f_{2}\left(e_{2} y\right)\right)$ is in $\left[l_{2}\left(\alpha_{2}+m_{2}\right), l_{2}\left(\alpha_{2}+n_{2}\right)\right]$, and $l_{3}\left(\alpha_{3}+f_{3}\left(e_{3} z\right)\right)$ is in $\left[l_{3}\left(\alpha_{3}+m_{3}\right), l_{3}\left(\alpha_{3}+n_{3}\right)\right]$. When 
$x, y, z$ are big enough, the three feedback functions cannot influence the chaotic nature of Rossler systems.

Let $a=0.05, b=10, c=0.2 ; l_{1}=l_{2}=40, l_{3}=1 ; \alpha_{1}=\alpha_{2}=\alpha_{3}=0.4 ; e_{1}=e_{2}=e_{3}=1$,

$f_{1}\left(e_{1} x\right)=\arccos \left(\cos \left(e_{1} x\right)\right), f_{2}\left(e_{2} y\right)=\arccos \left(\cos \left(e_{2} y\right)\right), f_{3}\left(e_{3} z\right)=\arccos \left(\cos \left(e_{3} z\right)\right)$. Then the attractors generated by system (2) are shown in figure 1.

Through the calculation, the Max Lyapunov Exponential (MLE) is $0.00657>0$, which means it is chaos. And from figure 3 we know that $z(t)$ has different positive levels.

\section{Dynamics analysis}

In this section, we will show that how the system behaviors change based on $l_{i}, \alpha_{i}, e_{i},(i=1,2,3)$.

From figure 3 we know that when the attractor moves to the left orbit of the striped attractor, the value of $z$ is almost zero. To be simple, we let $z \approx 0, z^{\prime} \approx 0$, and system (2) could be changed as:

$$
\left\{\begin{array}{l}
x^{\prime}=-y+l_{1}\left(\alpha_{1}+f_{1}\left(e_{1} x\right)\right) \\
y^{\prime}=x+a y+l_{2}\left(\alpha_{2}+f_{2}\left(e_{2} y\right)\right)
\end{array} .\right.
$$

and the stripe almost parallels to the axis $y$ at the left of attractor, then $x^{\prime} \approx 0$. So (3) can be replaced by

$$
y \approx l_{1}\left(\alpha_{1}+f_{1}\left(e_{1} x\right)\right) .
$$

Noting that $l_{1}\left(\alpha_{1}+f\left(e_{1} x\right)\right.$ is in $\left[l_{1}\left(\alpha_{1}+m_{1}\right), l_{1}\left(\alpha_{1}+n_{1}\right)\right]$, we have

$$
y \in\left[l_{1}\left(\alpha_{1}+m_{1}\right), l_{1}\left(\alpha_{1}+n_{1}\right)\right] .
$$

Similar to the above analysis, we can get that $x$ is roughly in $\left[-l_{2}\left(\alpha_{2}+n_{2}\right),-l_{2}\left(\alpha_{2}+m_{2}\right)\right]$, since $a$ is relatively small. Take $\alpha=0.216$ and $\alpha=0.4, f_{1}\left(e_{1} x\right)=\arccos \left(\cos \left(e_{1} x\right)\right)$,

$f_{2}\left(e_{2} y\right)=\arccos \left(\cos \left(e_{2} y\right)\right), f\left(e_{3} z\right)=\arccos \left(\cos \left(e_{3} z\right)\right)$. When $\alpha=0.216$, the length paralleled with axis $x$ is roughly in $[-139,-13]$, and the length paralleled with axis $y$ is roughly in $[13,139]$. When $\alpha=0.4$, they are roughly in $[-155,-29]$ and $[29,155]$, respectively.

When $l_{1}=l_{2}=0$, we can get that the length and the width of the rectangle are both zeros. In this case, we have figure 4. And figure 5 and 6 show the case of $l_{1}>0, l_{2}=0$ and $l_{1}=0, l_{2}>0$ respectively.

Now we turn to consider the connection between the shape and the parameter $e_{i}$. Let the period of $f_{1}(x), f_{2}(y)$ be $T_{1}, T_{2}$ respectively. Note that $f_{1}\left(e_{1} x\right)$ is a function with period $T_{1} / e_{1}$, which will keep the right-hand side of the first equation in system (2) unchanged when $x$ moves to $x \pm k T_{1} / e_{1}$ with some positive integer $k$. Similar discussion to $f_{2}\left(e_{2} y\right)$ can be done. As a result, if there are these parallel stripes, we have

$$
D_{\text {above }}=D_{\text {below }}=\frac{T_{2}}{e_{2}}, D_{\text {left }}=\frac{T_{1}}{e_{1}} .
$$

The result is shown in figure 7 when we take $f_{1}\left(e_{1} x\right)=\arccos (\cos (x)), f_{2}\left(e_{2} y\right)=\arccos (\cos (2 y))$. This may show the reason why $f$ needs to be selected as a periodic function.

\section{Conclusion}

This paper is studying some attractors and its property with continuous feedbacks, and finding some connections among the shapes, sizes, and parameters. After we get some connections among the shapes, sizes, and parameters, we validate them through some concrete data. The investigation for Rossler system has practical significance. However, nonlinear systems with noise are hard to be analyzed in a strict way; they deserve more attention in the future research on nonlinear dynamics.

\section{References}

Brown R, and Chua L O. (1996).Clarifying chaos: examples and counterexamples. J. of Bifurcation and Chaos. vol. 6 , 219-249.

Chen G, and Yu, eds. (2003). Chaos Control: Theory and Applications. Springer-Verlag.

Chen Q, Hong Y, Chen G, and Zhong Q. (2006). Chaotic attractors in striped rectangular shapes generated by a Rossler-like systems. Physics Letters A. vol. 348, 195-200. 
Chen Q, Zhang Y, and Hong Y. (2006). Generation and control of striped attractors of Rssler systems with feedback, Chaos, Solitons, and Fractals.

Gubckenheimer J and Holmes P. (1983). Nonlinear Oscillations, Dynamical Systems, and Bifurcations of Vector Fields. Springer-Verlag, New York.

Heagy J F, Platt N and Hammel S M. (1994). Characterization of on-off intermittency. Phys. Rev. E. 49(2), 1140-1150.

Hwang S, Gao J, and Liu J. (2000). Noise-induced chaos in an optically injected semiconductor laser model. Physical Review E. Vol. 61, 5162-5170.

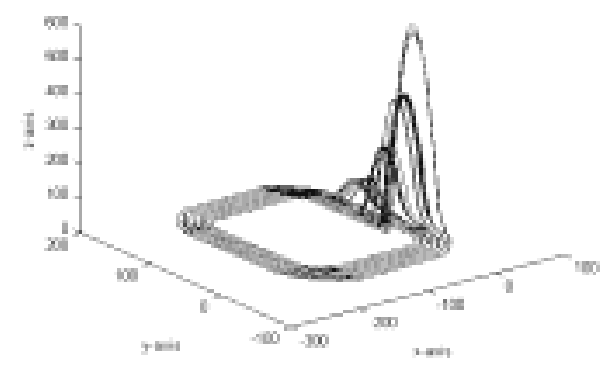

Figure 1. the attractor generated by system (2)

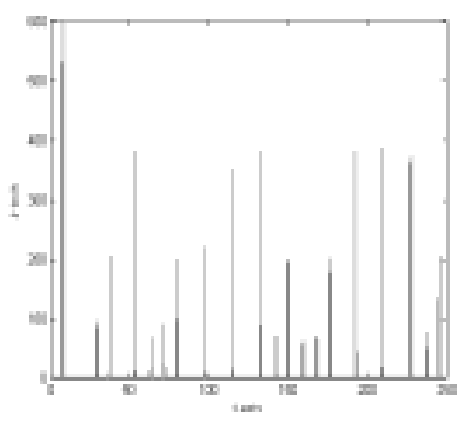

Figure 3. $t-z$ projections of the attractor

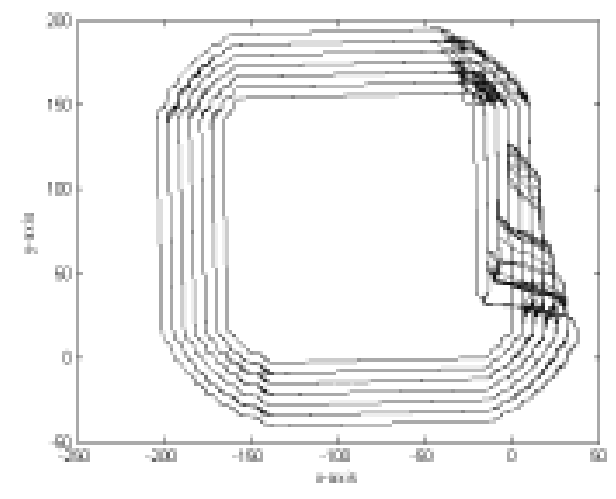

Figure 2. $x-y$ projections of the attractor

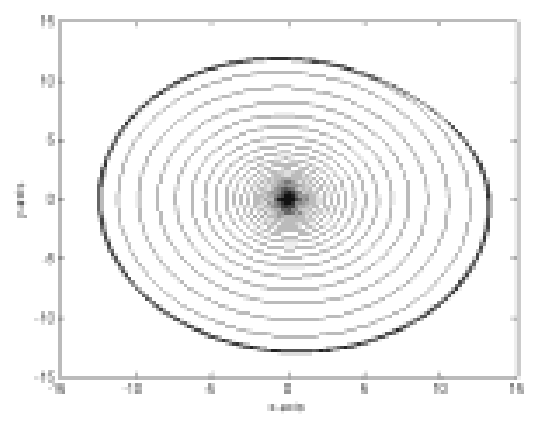

Figure $4 \cdot l_{1}=l_{2}=0, x-y$ projections of the attractor 


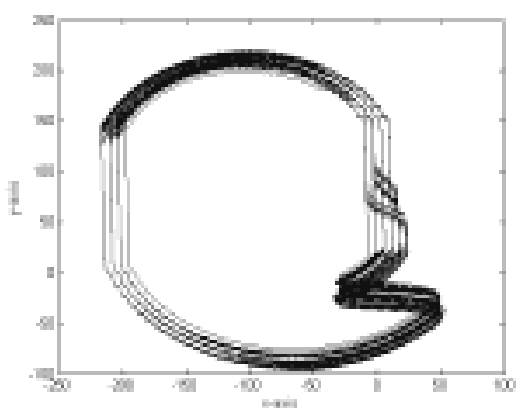

Figure 5. $l_{1}>0, l_{2}=0, x-y$ projections of the attractor

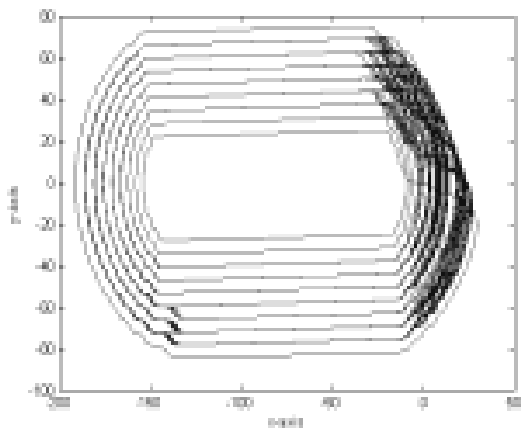

Figure 6. $l_{1}=0, l_{2}>0, x-y$ projections of the attractor

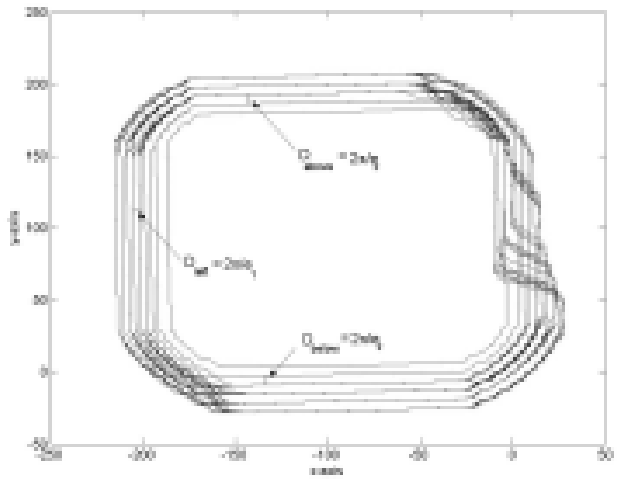

Figure 7. The $x-y$ projection with $e_{1}, e_{2}$ 